\title{
Nonlinear Observer Design for Guidance and Traction of Railway Vehicles
}

\author{
A. Heckmann, C. Schwarz, A. Keck, and T. Bünte \\ Institute of System Dynamics and Control, German Aerospace Center (DLR), \\ Münchener Str. 20, 82234 Wessling, Germany, \\ andreas.heckmann@dlr.de
}

\begin{abstract}
State observer design turned out to be crucial in several recent railway vehicles projects on active control and condition monitoring at DLR, but may also be useful for emerging technologies like the cyber physical system and the digital twin approach or the realization of predictive maintenance concepts. With this background and motivation the paper presents a process in three steps: (i) an initial analysis results in a physical model, (ii) the subsequent transfer to state space representation facilitates the prove of observability and (iii) the observer synthesis supports the design of the observer feedback law. Results from two projects, one related to longitudinal or traction dynamics, the other associated to the guidance task of independently rotating wheels, demonstrate the application of the observer design process and offers a comparison of observer estimates with measurements.
\end{abstract}

Keywords: State observer, railway vehicles, traction, guidance

\section{Background and Motivation}

State observer design is a task that turned out to be crucial in several recent railway vehicles projects on active control and condition monitoring at DLR such as the Next Generation Train (NGT) [1] and the DynORail [2] project. Moreover, there are a number of existent or emerging vehicle technologies that rely on the capability to gain, process and communicate sensor data: (i) Active control of railway vehicles offers safety and comfort enhancements and allows for reducing energy consumption and wear. (ii) Condition monitoring and predictive maintenance promise to reduce life-cycle costs and to improve the operational availability or reliability, respectively. (iii) The cyber physical system approach aims at benefits due to real-time communication and integration of vehicles with their environments. (iiii) The digital twin technology enables optimization and engineering of vehicle systems in operational and retrofit scenarios and pledge high quality real life data for future designs of vehicle components.

All of these technologies extensively use measurements and there again is a multitude of methods that might be used to analyse and process this data, whereas artificial intelligence and data sciences have gained popularity, recently. However, the traditional concept of a state observer exhibits several outstanding 
advantages: (i) An observer provides access to information which otherwise is not readily available or at high cost, only. (ii) It is capable of exploiting expert knowledge by means of a physical system description. (iii) Observer results may be retraced and physically interpreted. (iiii) The stability and robustness of the observer dynamics can be assessed, which reduces posssible restrictions of the further use of its outputs e.g. regarding feedback control.

With this background and motivation the paper proposes a design process of a nonlinear observer in three steps in the following section. This design process is then exemplified for an application in longitudinal dynamics or traction, respectively, in Sec. 3 and for lateral dynamics or guidance in Sec. 4. The final section provides a summary and an outlook.

\section{Overview of the Design Procedure}

\subsection{Physical System Analysis}

It is a commonplace that all modeling begins with the determination of the actual system of interest. This fundamental task includes the definition of the boundaries that separate the system from its environment and the physical quantities that flow aross these borders and organize the interaction of the system with its surroundings.

As regards railway vehicle dynamics, the system could contain e.g. the complete train, a single car, a running gear or a wheel, only. Its dynamics could include six translational and rotational degrees of freedom or may be confined to a subset. In fact, confinement according to the axiom "Everything should be made as simple as possible, but not simpler" ${ }^{1}$ presents the actual goal of physical system analysis in order to gain a model that is intended to tackle the engineering problem on hand.

In this work, we propose two separate observers, one for longitudinal running gear dynamics in Sec. 3 and one for lateral dynamics in Sec. 4. Detailed analysis reveals that longitudinal and lateral dynamics are only weakly coupled in case of small slip, see the linearized analytical model of a running gear $[3,(5)]$, which still exhibits this coupling. As a consequence for the synthesis of a guidance controller in $[3$, Sec. 3.3], it is reasonable to neglect longitudinal wheel slip. The same reasoning applies to observer design. On the other hand, the lateral dynamics has a much lower impact on longitudinal dynamics than the wheel-rail friction conditions and can therefore be neglected.

\subsection{State Space Representation}

Once a physical model is available, it has to be transformed into a mathematical form in order to systematically apply and optionally extend analysis and

\footnotetext{
${ }^{1}$ attributed to Albert Einstein, but possibly paraphrased, see https://en.wikiquote. org/wiki/Albert_Einstein
} 
synthesis methods from literature and prevent "the reinvention of the wheel":

$$
\dot{\hat{\boldsymbol{x}}}=\hat{\boldsymbol{f}}(\hat{\boldsymbol{x}}, \boldsymbol{u}, v), \quad \hat{\boldsymbol{y}}=\hat{\boldsymbol{g}}(\hat{\boldsymbol{x}}, \boldsymbol{u}, v),
$$

where $\hat{\boldsymbol{x}}$ denotes the estimate of $\boldsymbol{x}$, which is the unknown state vector of the real life system. The vector $\boldsymbol{u}$ represents the system input,the longitudinal speed $v$ is a parameter that significantly influences the dynamics of the system and considered to be known, see [3]. The prove of system observability is a prominent analysis method to be applied to (1).

Note that the in general unknown real life system

$$
\dot{\boldsymbol{x}}=\boldsymbol{f}(\boldsymbol{x}, \boldsymbol{u}, v)+\boldsymbol{\mu}, \quad \boldsymbol{y}=\boldsymbol{g}(\boldsymbol{x}, \boldsymbol{u}, v)+\boldsymbol{\rho},
$$

includes process and measurement noise contributions $\boldsymbol{\mu}$ and $\boldsymbol{\rho}$, which are assumed to be additive and Gaussian with zero mean.

\subsection{Observer Synthesis}

The construction of the rule $\boldsymbol{L}(\hat{\boldsymbol{x}}, \boldsymbol{u}, v)$ used to feed back the output error constituted by comparison of the observer outputs $\hat{\boldsymbol{y}}$ and the measurements $\boldsymbol{y}$ constitutes the third step of the design process [4]:

$$
\dot{\hat{\boldsymbol{x}}}=\hat{\boldsymbol{f}}(\hat{\boldsymbol{x}}, \boldsymbol{u}, v)+\boldsymbol{L}(\hat{\boldsymbol{x}}, \boldsymbol{u}, v)(\boldsymbol{y}-\hat{\boldsymbol{y}}), \quad \hat{\boldsymbol{y}}=\hat{\boldsymbol{g}}(\hat{\boldsymbol{x}}, \boldsymbol{u}, v) .
$$

In the simplest case related to the linear Luenberger Observer [4, Sec. 7.2], $\boldsymbol{L}$ may denote a constant feedback matrix whose entries are determined by offline numerical optimization using available measurements, see [2].

More sophisticated synthesis methods follow the idea of the Kalman Filter and explicitly consider $\boldsymbol{\mu}$ and $\boldsymbol{\rho}$ or the associated covariance matrices $\boldsymbol{Q}$ and $\boldsymbol{R}$, respectively, [4]. For nonlinear systems as well, several algorithms such as the Extended or the Unscented Kalman Filter exist that provide estimates of the current state variables along with their uncertainties, cf. [5].

With this background, the specific synthesis task consists of the design of $\boldsymbol{Q}$ and $R$ in such a way that the convergence of the estimation error is ensured. Provided that real life measurement data $\boldsymbol{y}$ is available, numerical optimization of the following problem with multiple cases $S_{k} \in S$, which cover the relevant application scenarios, is an appropriate tool to do so:

$$
\min _{\nu} \max _{k \in S} o_{k}(\nu), \quad \nu:=\operatorname{diag}([\operatorname{diag}(\boldsymbol{Q}), \operatorname{diag}(\boldsymbol{R})]), \quad o_{k}:=\frac{1}{t_{k}} \int_{0}^{t_{k}}|\boldsymbol{y}-\hat{\boldsymbol{y}}| \mathrm{d} t .
$$

\section{Traction}

\subsection{Physical Model}

Here, the term traction is used to cover phenomena associated to both, accelerating and decelerating or driving and braking forces that act at the wheel-rail 


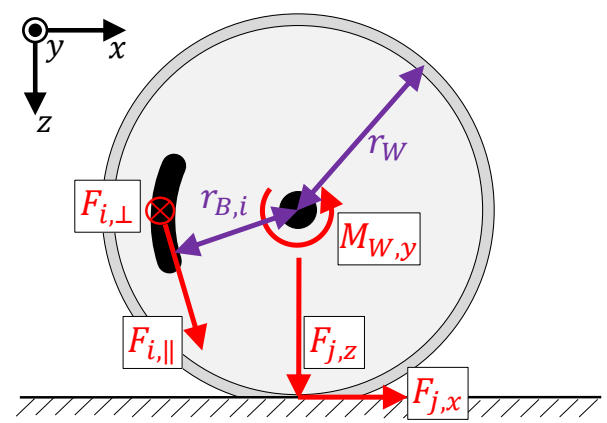

Fig. 1. Free body sketch of a wheelset, applied forces and important parameters

interface. Its adhesion conditions are characterized by the adhesion or currently exploited friction coefficient $\mu_{W R}$. In friction braking scenarios, the instantaneous friction coefficient $\mu_{D C}$ between brake disc and pad surface is additionally significant. Since these friction or adhesion coefficients, respectively, are highly safety relevant, exhibit large fluctuations, but cannot be measured directly, it is intended to design an observer that is capable of providing this information online in operation.

In order to balance computational requirements, accuracy and system dimensions the system in Fig. 1 is proposed for observer design. The associated equations that rule the rotational motion of the wheelset $\omega_{W}$ read

$$
\begin{aligned}
J_{W, y} \cdot \dot{\omega}_{W} & =\sum_{i} F_{i, \|} \cdot r_{B, i}+\sum_{j} F_{j, x} \cdot r_{W, j}+M_{W, y}, \\
F_{i, \|} & =\mu_{D C, i} \cdot F_{i, \perp}=\mu_{D C, i} \cdot F_{i, \perp}\left(p_{B}\right), \\
F_{j, x} & =\mu_{W R, j} \cdot F_{j, z} .
\end{aligned}
$$

The index $i$ identifies each brake unit, while $j=l, r$ distinguishes between quantities on the left or right wheel. $M_{W, y}$ summarizes all torques applied by the attached power train. $p_{B}$ denotes the pneumatic pressure of the brake actuators that apply forces $F_{i, \perp}$ normal to the brake discs.

A survey on the parameter variations in operation revealed that the wheel radius $r_{W}$, the brake radius $r_{B, j}$ and even the wheel load $F_{j, z}$ vary only little in comparison to the friction or adhesion coefficients, so that they may be assumed to be constant [2]. The assumptions $\mu_{W R, l} \approx \mu_{W R}, \mu_{W R, r} \approx \mu_{W R}$ and $\mu_{D C, i} \approx$ $\mu_{D C}$ enable a further simplification of the description. In other words: it is neither intended or possible, respectively, to independently observe the friction conditions on both wheels nor at different brake discs.

A rolling contact description from literature [6] supplements the physical description of the model in Fig.1, where nominal values of geometrical and physical contact parameters are wrapped up to get two quantities $A$ and $B$ and 
to define a nominal value of $\mu_{W R}$, namely

$$
\mu_{W R, 0}=A\left(\frac{\varepsilon}{1+\varepsilon^{2}} \arctan (\varepsilon)\right), \quad \varepsilon:=B \cdot \frac{v+\omega_{W} \cdot r_{W}}{v} .
$$

In the same manner, a relationship may be introduced to physically motivate $\mu_{D C}$ e.g. as a function of the brake disc temperature, but we assume the nominal coefficient $\mu_{D C, 0}$ to be constant.

\subsection{State Space Representation}

As already prepared, the friction terms are separated into a nominal contribution and a fluctuation term intended to be observed:

$$
\mu_{W R}=\mu_{W R, 0}+\Delta \mu_{W R}, \quad \mu_{D C}=\mu_{D C, 0}+\Delta \mu_{D C} .
$$

The estimate of the state vector $\hat{x}$, the input vector $\boldsymbol{u}$ and the measurement vector $\boldsymbol{y}$ to be introduced into (1) using (5) and (6) then read

$$
\hat{\boldsymbol{x}}=\left(\begin{array}{c}
\hat{\omega}_{W} \\
\hat{v} \\
\Delta \hat{\mu}_{D C} \\
\Delta \hat{\mu}_{W R}
\end{array}\right), \quad \boldsymbol{u}=\left(\begin{array}{c}
p_{B} \\
M_{W, y}
\end{array}\right), \quad \boldsymbol{y}=\left(\begin{array}{c}
\omega_{W} \\
v
\end{array}\right)
$$

A nonlinear analysis of (1) with (7) proved the system to be locally observable, i.e. the system is observable presuming that $p_{B} \neq 0$ and $v \neq 0$.

\subsection{Observer Synthesis and Exemplary Results}

The synthesis process in order to design the feedback law $\boldsymbol{L}$ in (2) for the system in (7) is presented in Sec. 2.3.

Fig. 2 presents corresponding results in the sequel to the DynORail project [2], where the adhesion conditions were measured on a true scale roller rig. The observer, an Extended Kalman Filter [4, Sec.7.4.2], here was operated offline, but fed with $\boldsymbol{u}=\boldsymbol{u}(t)$ as given in (7), while $v$ in $\boldsymbol{y}=\boldsymbol{y}(t)$ was substituted by the angular roller speed $\omega_{R}$ using its radius $r_{R}$, i.e. $v=\omega_{R} \cdot r_{R}$

Shown is a challenging scenario under wet conditions that forces the wheelslide protection system to interfere after roughly $8 \mathrm{~s}$. The measured wheel-rail adhesion coefficient and the estimated values of $\mu_{W R}$ significantly differ only at the very beginning of the scenario or braking maneuver, respectively, where the observer takes some time to tune up. The corresponding measurements of $\mu_{D C}$ were not available and could therefore not be plotted in Fig. 2, but the observer estimate appears to be sound and reasonable. 


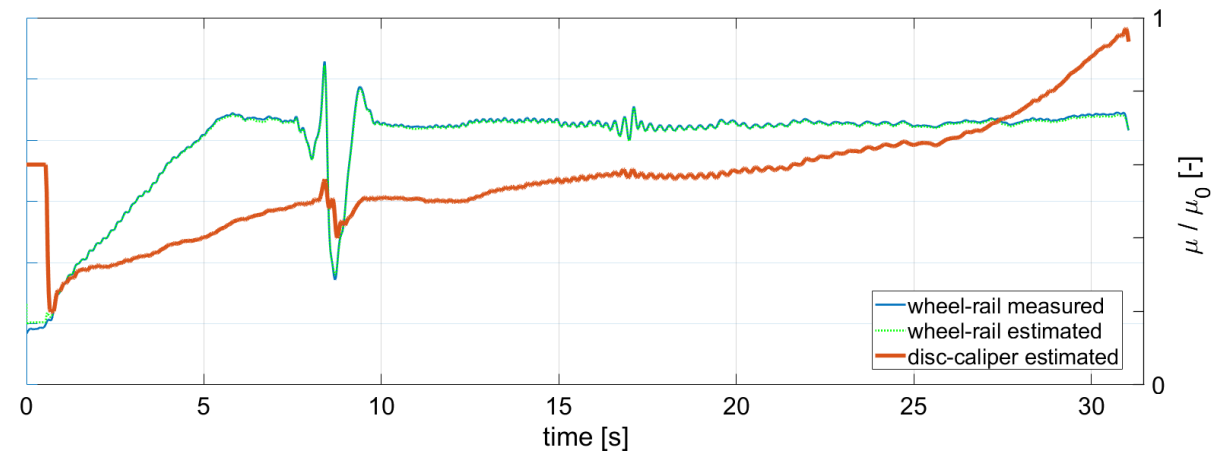

Fig. 2. Comparison of measured and estimated results for $\mu_{W R}$ and estimation of $\mu_{D C}$

\section{Guidance}

\subsection{Physical Model}

The NGT project addresses the development of a running gear that makes axle shafts obsolete but relies on active control. Fig. 3 presents an experimental running in scale 1:5, which is operated as hardware test bench in order to support research activities on observer and control design.

The running gear is equipped with four independently rotating wheels (IRW) each driven by an in-wheel motor. Each motor is controlled by its own power converter. The axle bridges are connected to the running gear frame via a leaf spring guidance. The mounting and the vertical spring represent the primary suspension of the running gear. This configuration enables a yaw motion of the axle bridges, that is limited by bump stops. The central frame is mounted to the roller rig with a lemniscate guidance, which locks the longitudinal motion.

Fig. 3 displays the entire degrees of freedom of the running gear. These are the four wheel rotations $\omega_{m s}$ with $m=f$ (front), $r$ (rear) and $s=l$ (left), $r$ (right), the yaw motions of the wheel-carriers $\psi_{m}$ and the three rotational as well as the vertical and lateral motions of the central frame $\alpha_{F}, \beta_{F}, \psi_{F}, z_{F}$, and $y_{F}$, respectively. For detailed description see [7], where the equations of motion are given as well.

In terms of a model-based control design, the controller performance highly depends on the estimated lateral positions $y_{f}$ and $y_{r}$ of the running gear relative to the center line of the track [1]. However under real life conditions at railway tracks, these lateral positions are difficult to measure in a reliable and robust manner. This is the motivation to develop an observer for $y_{f}$ and $y_{r}$.

\subsection{State Space Representation}

The reformulation of the equations of motion of a single axle configuration in [7] leads to a system according to (1) [8]. However, the analysis process revealed two options to model the system. The more elaborate initial system assumes 


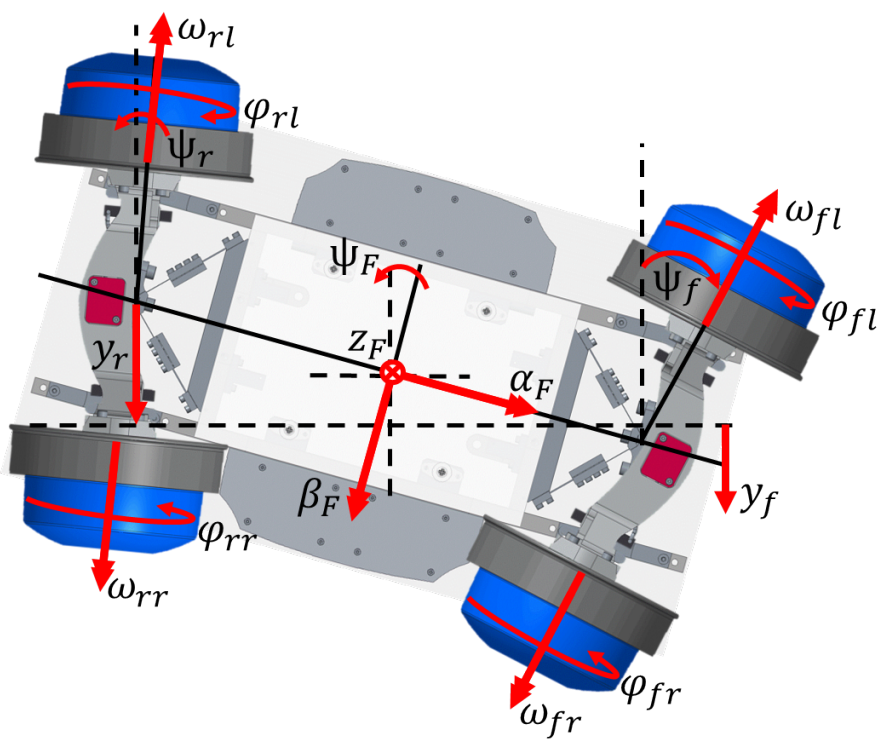

Fig. 3. Scaled experimental running gear at DLR

the state vector $\hat{\boldsymbol{x}}_{6}$, while the reduced state vector $\hat{\boldsymbol{x}}_{5}$ could be defined if the longitudinal wheel slip is neglected assuming $y_{f} \sim v \psi_{f}$ :

$$
\hat{\boldsymbol{x}}_{5}=\left[y_{f}, \psi_{f}, \dot{\psi}_{f}, \omega_{f r}, \omega_{f l}\right]^{T}, \quad \hat{\boldsymbol{x}}_{6}=\left[y_{f}, \dot{y}_{f}, \psi_{f}, \dot{\psi}_{f}, \omega_{f r}, \omega_{f l}\right]^{T} .
$$

It is an important objective of research to examine several alternative sensor configurations and tell their properties. That is why the following three sensor compositions are taken under consideration:

$$
\begin{gathered}
\hat{\boldsymbol{g}}_{3}=\left[\psi_{f}, \omega_{f r}, \omega_{f l}\right]^{T}, \quad \hat{\boldsymbol{g}}_{5}=\left[\psi_{f}, \omega_{f r}, \omega_{f l}, \ddot{y}_{f}, \dot{\psi}_{f}\right]^{T}, \\
\hat{\boldsymbol{g}}_{6}=\left[\psi_{f}, \omega_{f r}, \omega_{f l}, F_{y}, F_{z}, M_{x}\right]^{T} .
\end{gathered}
$$

The low-level set-up $\hat{\boldsymbol{g}}_{3}$ consists of the yaw angle $\psi_{f}$ and the rotational speed $\omega_{f s}$ signals of the front wheel-pair. This configuration is extended in the mid-level setup $\hat{\boldsymbol{g}}_{5}$ signals $\ddot{y}_{f}$ and $\dot{\psi}_{f}$ provided by an inertia measurement unit (IMU) at the center of the axle bridge. The high-level design $\hat{\boldsymbol{g}}_{6}$ is generated through the combination of $\hat{\boldsymbol{g}}_{3}$ and the resultant forces $F_{y}$ and $F_{z}$ and the resultant torque $M_{x}$, acting on the axle bridge and provided by force and torque sensors (FTS) mounted at the wheel bearings.

A rigorous nonlinear analysis showed that the set-up $\hat{\boldsymbol{g}}_{3}$ only guarantees local observability for the reduced system $\hat{\boldsymbol{x}}_{5}$, which however might not be valid during acceleration or deceleration processes with high slip conditions [8]. The full order 
system $\hat{\boldsymbol{x}}_{6}$ is observable with both $\hat{\boldsymbol{g}}_{5}$ and $\hat{\boldsymbol{g}}_{6}$, but as well relies on $v \neq 0$, which implies local observability.

Eqs. (9) may also be used to analyze the expected estimation error $\Delta y_{f}$, which follows from the specified sensor errors and their propagation through the measurement equations. Table 1 presents results of this analysis for the M 1:5 experimental running gear at DLR.

\begin{tabular}{|c|c|c|}
\hline configurations & $\begin{array}{c}\Delta y_{f} \text { under M 1:5 laboratory } \\
\text { conditions }\end{array}$ & $\begin{array}{c}\Delta y_{f} \text { under M 1:1 real life } \\
\text { conditions }\end{array}$ \\
\hline$\hat{\boldsymbol{x}}_{5}, \hat{\boldsymbol{g}}_{3}$ & $\approx 5 \cdot 10^{-4} \mathrm{~m}$ & $\approx 8.5 \cdot 10^{-4} \mathrm{~m}$ \\
\hline$\hat{\boldsymbol{x}}_{6}, \hat{\boldsymbol{g}}_{5}$ & $\approx 1.4 \cdot 10^{-4} \mathrm{~m}$ & $\approx 5.3 \cdot 10^{-4} \mathrm{~m}$ \\
\hline$\hat{\boldsymbol{x}}_{6}, \hat{\boldsymbol{g}}_{6}$ & $\approx 1.2 \cdot 10^{-4} \mathrm{~m}$ & $\approx 4.5 \cdot 10^{-4} \mathrm{~m}$ \\
\hline
\end{tabular}

Table 1. Expected estimation error $\Delta y_{f}$ due to the propagation of sensor errors for three different configurations and two different sets of sensors.

A preliminary survey on available sensors for industrial use resulted in following sensor errors that may be attainable under M 1:1 real life conditions:

$$
\begin{aligned}
& \Delta \omega_{m s} \approx 2.4 \cdot 10^{-5} \mathrm{rad} / \mathrm{s}, \quad \Delta \ddot{y}_{m s} \approx 2.4 \cdot 10^{-3} \mathrm{~m} / \mathrm{s}^{2}, \quad \Delta F_{y} \approx 2.5 \mathrm{~N} \text {, } \\
& \Delta \psi_{m} \approx 1.9 \cdot 10^{-4} \mathrm{rad}, \quad \Delta \dot{\psi}_{m} \approx 6.5 \cdot 10^{-5} \mathrm{rad} / \mathrm{s}, \quad \Delta M_{x} \approx 0.5 \mathrm{Nm} \text {. }
\end{aligned}
$$

Table 1 as well presents the expected estimation error $\Delta y_{f}$ that follows from these sensor errors. Note that the observer actually does not rely on absolute values but on relative fluctuations of the measurement signals. Nevertheless, the requirements in particular for the FTS under real life conditions are challenging, so that the sensor configuration $\hat{\boldsymbol{g}}_{5}$ might be more appropriate than $\hat{\boldsymbol{g}}_{6}$ in order to balance accuracy and economic aspects.

\subsection{Observer Synthesis and Exemplary Results}

Here, the covariance matrices $\boldsymbol{Q}$ and $\boldsymbol{R}$ are optimized for two different filter algorithms, for the Extended Kalman Filter (EKF) and the Unscented Kalman Filter (UKF). The multi-case optimization set included 27 different motion scenarios, which reproduce the natural hunting motion of conventional wheelsets with three different lateral amplitudes, three running speeds $v$ and three hunting frequencies, but are driven by active control of the IRW, see [8].

Fig. 4 shows selected results of the optimization process in the simulation environment. The error tolerance bands are plotted for $\hat{\boldsymbol{g}}_{3}$ (below green line) and $\hat{\boldsymbol{g}}_{6}$ (below gray line). The estimated lateral positions of both observers are within the corresponding bounds, which indicates that the observer design process was successful at least for the chosen motion cases of the running gear. Fig. 4 also shows that the estimation errors of the UKF are $50 \%$ the EKF estimations. 


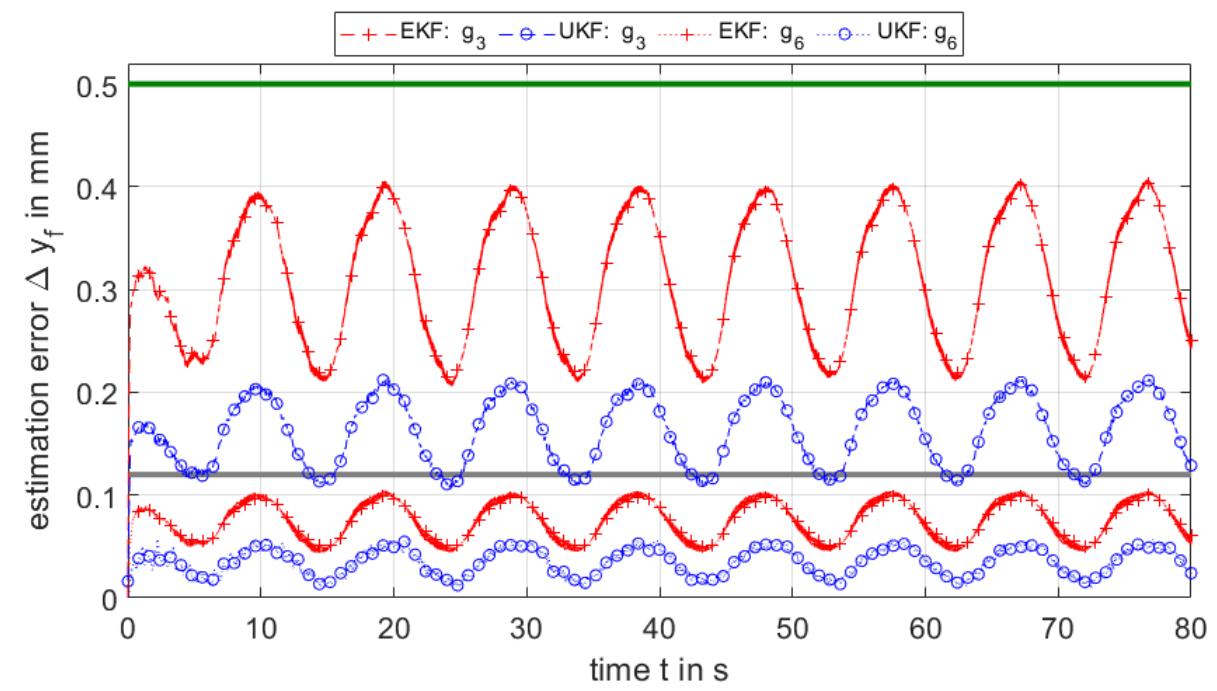

Fig. 4. Estimation error for different observer settings as obtained by comparison to $y_{f}$ measurements at the M1:5 experimental running gear.

\section{$5 \quad$ Summary and Outlook}

The paper presents a state observer design process in three steps and demonstrate its application in two cases: the first application concerns the estimation of the adhesion conditions of a wheelset at the wheel-rail and the brake-pad interface during braking, while the second example is associated to the appraisal of the lateral position of an IRW wheel pair with respect to the center line of the track. Both observers are validated by comparison of observer estimates with measurement data.

An accompanying paper [9] addresses the control of the IRW wheel pair of the NGT running gear and uses a specific approach to consider in particular the nonlinear wheel-rail profile geometry. This approach has already been adapted in the theoretical, analytical and simulation work associated to the guidance observer design. Future work will tackle implementation and experimental validation at the rapid control prototyping environment of the scaled experimental running gear at DLR .

\section{Acknowledgment}

The DynORail project was supported by StMWi (StMWi grant number: MST1308-0006// BAY 191/002), the Bavarian Ministry of Economic Affairs and Media, Energy and Technology. We very much appreciate the provision of roller rig measurement data by Knorr Bremse SfS, Munich, in the course of this project. 


\section{References}

1. A. Heckmann, D. Lüdicke, G. Grether, and A. Keck. From scaled experiments of mechatronic guidance to multibody simulations of DLR's Next Generation Train set. In M. Spiryagin, T. Gordon, C. Cole, and T. McSweeney, editors, 25th International Symposium on Dynamics of Vehicles on Roads and Tracks, Dynamics of Vehicles on Roads and Tracks. CRC Press, 2017.

2. C. Schwarz, J. Brembeck, and B. Heckmann. Dynamics observer for the longitudinal behavior of a wheelset on a roller rig. Proceedings of the Institution of Mechanical Engineers, Part F: Journal of Rail and Rapid Transit, 2018.

3. A. Heckmann, C. Schwarz, T. Bünte, A. Keck, and J. Brembeck. Control development for the scaled experimental railway running gear of DLR. In M. Rosenberger, M. Plöchl, K. Six, and J. Edelmann, editors, 24th Symposium of the International Association for Vehicle System Dynamics (IAVSD 2015), volume 1 of The Dynamics of Vehicles on Roads and Tracks. CRC Press 2016, 2016.

4. J. Adamy. Nichtlineare Systeme und Regelungen. Springer, 2018.

5. J. Brembeck. Nonlinear constrained moving horizon estimation applied to vehicle position estimation. Sensors, 19(10):2276, 2019.

6. O. Polach. Creep forces in simulations of traction vehicles running on adhesion limit. Wear, 258(7):992-1000, 2005.

7. C. Schwarz, A. Heckmann, and A. Keck. Different models of a scaled experimental running gear for the DLR RailwayDynamics Library. In 11th International Modelica Conference, 21.-23. Sep. 2015, 2015.

8. A. Keck, C. Schwarz, and T. Meurer. Observer design for a railway running gear with independently rotating wheels. In Joint 8th IFAC Symposium on Mechatronic Systems and 11th IFAC Symposium on Nonlinear Control Systems, 2019.

9. G. Grether, G. Looye, and A. Heckmann. Lateral guidance control using information of preceding wheel pairs. In Proceedings of the 26th International Symposium on Dynamics of Vehiles on Roads and Tracks, IAVSD 2019, 2019. Accepted for publication. 